\section{Buchrezension zu: Heilpflanzenlexikon}

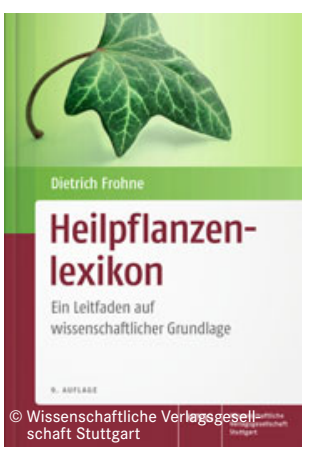

Heilpflanzenlexikon

Ein Leitfaden auf wissenschaftlicher Grundlage

Dietrich Frohne

686 S., Wissenschaftliche

Verlagsgesellschaft Stuttgart, 9. Aufl., 2021. $H C, 54,00 €$.

ISBN: 9783804737006

DOI: $10.1007 / \mathrm{s} 12268-021-1628-y$

(C) Der Autor 2021

Das allgemeine gesellschaftliche Interesse an der Naturheilkunde ist riesengroß. Pflanzliche („,biologische“) Arzneimittel gelten als gesund, während synthetische („chemische“) Arzneimittel keinen guten Ruf genießen. Dabei ist diese weitverbreitete Annahme Unsinn, denn auch die Inhaltsstoffe von pflanzlichen Arzneimitteln sind chemische Verbindungen. Diese Irrtümer zeigen schlaglichtartig gravierende naturwissenschaftliche Bildungsdefizite in weiten Teilen der Bevölkerung auf.

Ein klassisches Nachschlagewerk über Heilpflanzen ist „der Frohne“, der 2006 in seiner 8. Auflage erschien. Inzwischen sind 15 Jahre vergangen, aber das Interesse an dem Thema ist ungebrochen. Dies erkennt man daran, dass die 8. Auflage inzwischen vergriffen ist; aber die Nachfrage ist immens. Diese Ausgangssituation veranlasste den Verlag dazu, eine 9. Auflage des Lexikons auf den Markt zu bringen. Da der Autor im Jahr 2017 verstarb und offensichtlich kein Nachfolger für die Weiterbetreuung des Lexikons gefunden wurde, beschränkte sich der Verlag auf eine
Neuauflage, in der vor allem das Layout modernisiert wurde.

Dies bedeutet, dass das Buch inhaltlich auf einem Wissenstand von vor ca. 20 Jahren ist. Es werden alphabetisch viele bekannte (und weniger bekannte) Heilpflanzen hinsichtlich verwendeter Pflanzenteile, Inhaltsstoffen, Anwendungen und unerwünschter Wirkungen beschrieben. Leider fehlen in den meisten Fällen Darstellungen zum molekularen Wirkmechanismus der Inhaltsstoffe von Heilpflanzen. Auch werden Anwendungen von Heilpflanzen nicht nach den Prinzipien der evidenzbasierten Medizin dargestellt, sondern nach der „Erfahrungsmedizin“. Etliche im Lexikon empfohlene Anwendungen sind sogar gefährlich und nicht mehr zeitgemäß wie z. B. die Gabe von Ipecacuanhaextrakt zur Auslösung von Erbrechen. Andererseits fehlt eine Darstellung moderner Anwendungen von Inhaltsstoffen aus Arzneipflanzen, wie die Gabe von Colchicin bei der Perikarditis. In gewisser Weise beleuchtet das Lexikon „negativ“ den wissenschaftlichen Fortschritt auf dem Gebiet der Heilpflanzen, den es in den letzten 20 Jahren gegeben hat.

Das Buch ist vor allem etwas für Liebhaber und Buchsammler mit einer Schwäche für die $\mathrm{Ge}$ schichte der Heilpflanzen. Es werden veraltete Begriffe verwendet, die nicht mehr moderner pharmakologischer Nomenklatur entsprechen. Das Buch ist definitiv nicht dazu geeignet, sich auf den neuesten wissenschaftlichen Stand zum Thema Arzneistoffe aus Heilpflanzen zu bringen. Dazu wäre eine fundamentale Überarbeitung notwendig. Der Preis ist recht hoch für ein Werk, in dem leider keine der vielen (schön anzusehenden, aber teilweise auch giftigen) Heilpflanzen illustriert werden. Aus Sicht des Rezensenten ist es also ein Auslaufmodell, aber für einen interessierten pharmazeutischen Biologen er- gibt sich, das zeigt der Büchermarkt, eine große Spielwiese.

Roland Seifert,

Medizinische Hochschule Hannover

seifert.roland@mh-hannover.de

Diese Rezension erscheint Open Access. ${ }^{*}$

* Funding note: Open Access funding

enabled and organized by Projekt DEAL. Open Access: Dieser Artikel wird unter der Creative Commons Namensnennung $4.0 \mathrm{In}$ ternational Lizenz veröffentlicht, welche die Nutzung, Vervielfältigung, Bearbeitung, Verbreitung und Wiedergabe in jeglichem Medium und Format erlaubt, sofern Sie den die ursprünglichen Autor(en) und die Quelle ordnungsgemäß nennen, einen Link zur Creative Commons Lizenz beifügen und angeben, ob Änderungen vorgenommen wurden. Die in diesem Artikel enthaltenen Bilder und sonstiges Drittmaterial unterliegen ebenfalls der genannten Creative Commons Lizenz, sofern sich aus der Abbildungslegende nichts anderes ergibt. Sofern das betreffende Material nicht unter der genannten Creative Commons Lizenz steht und die betreffende Handlung nicht nach gesetzlichen Vorschriften erlaubt ist, ist für die oben aufgeführten Weiterverwendungen des Materials die Einwilligung des jeweiligen Rechteinhabers einzuholen. Weitere Details zur Lizenz entnehmen Sie bitte der Lizenzinformation auf http://creativecommons.org/ licenses/by/4.0/deed.de. 